\title{
Universal mathematical model of SAR signals for natural surfaces
}

\author{
Alexander Bokov*, Yury Markov, Vladimir Vazhenin, and Sergey Margilevsky \\ Ural Federal University, Yekaterinburg, Russian Federation
}

\begin{abstract}
The implementation of the basic version of the universal mathematical model of the formation of reflected signals for various airborne radar systems, including the synthetic antenna aperture radar, when working on natural surfaces are discussed in the article. A number of parameters are required to study, which must be taken into account for the correct modeling of echo signals, which must then be processed to calculate radar images. A method for constructing such a mathematical model in MATLAB is presented with some modeling results for a homogeneous rough surface and two typical pulse and continuous modulation signals. But the facet parameter structure of the simulated surface can take into account the possibilities of representing various types of terrain and objects created by man.
\end{abstract}

\section{Introduction}

To develop and improve Earth remote sensing methods, mathematical modeling of the processes of radiation, propagation, reflection, or, in other words, scattering, reception and processing of radar signals is required [1-3]. Modern computing resources make it possible to implement ever more detailed and accurate mathematical models, but in practice they are limited to design specialized models for a particular type of system, type of signal, type of surface, and method for processing and synthesizing radar images [4-6].

The article covers the topic of the implementation of the basic version of a universal mathematical model for a research software package designed to test and evaluate the quality indicators of the algorithms for the operation of radars with synthetic antenna aperture (SAR) in various application conditions. The software package is built from a set of modules combined into a system for modeling reflected signals for a given trajectory of movement, the orientation of the aircraft (as the radar platform) and the characteristics of the underlying surface.

\section{Surface modelling}

A mathematical model of the surface strongly depends on type of terrain, as well as on possibilities of accounting all the properties of physical process of electromagnetic waves reflection $[2,7]$. For the mathematical description of the natural earth's surface, the facet surface model is justified and selected, which is the most universal for various types of

\footnotetext{
* Corresponding author: a.s.bokov@urfu.ru
} 
point, surface-distributed and volume-distributed reflectors of radar targets/terrain [1-5]. It is based on a geometric model of propagation and reflection of radio waves from the entire set of trained reflectors, which are presented in the form of conditional facets, i.e. simplified flat areas with dimensions less than the radar resolution. Each $i^{\text {th }}$ facet has a biased backscattering pattern. But the form of a backscattering pattern is equal for the same type of material (substance) of reflecting surface.

A typical geometric model of a radio altimeter operation with a radar carrier platform that moves over the facet field, which represents a set of simulated reflectors, is shown in Fig. 1. The typical forms of an altimeter antenna pattern, illuminated footprint and backscattering pattern of a rough surface are also presented there. In the practical case, when the carrier platform moves, the footprint spot also moves with the same speed and direction. Moreover, the radar antenna (and/or its main lobe) may be biased due to the aircraft evolutions or the antenna scanning system. Thus, the simulated surface should be expanded taking into account the entire flight path and angle evolutions of the antenna main lobe.

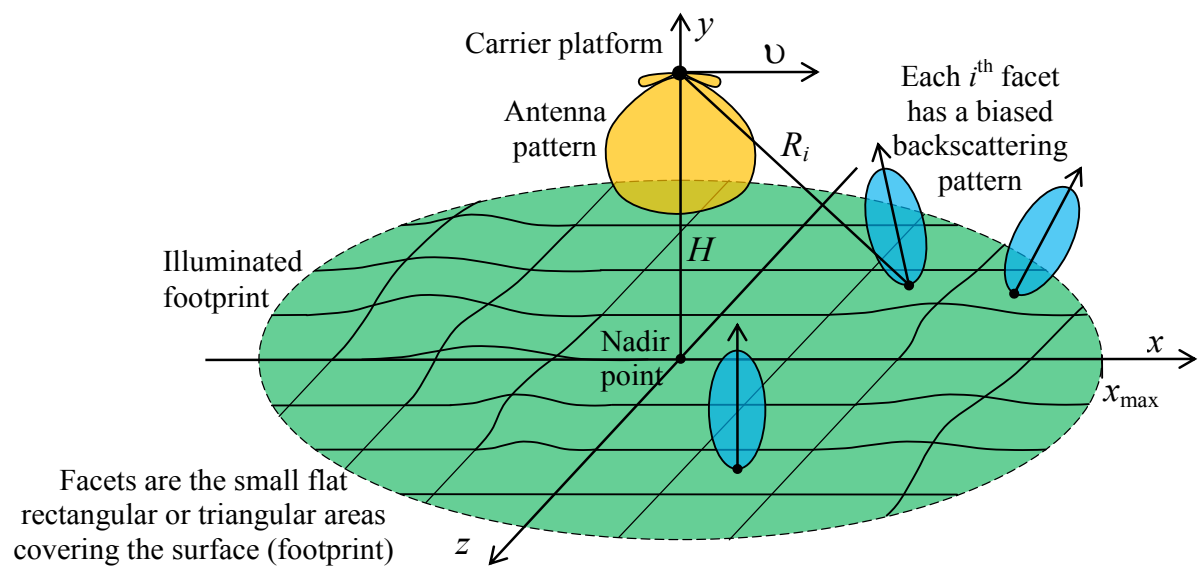

Fig. 1. Radar scene with facet field for modeling reflectons from the underlying surface.

The modeling method used in the work involves the joint use of the phenomenological approach and the totality of empirical data. In the framework of this approach, the facets of the irradiated surface are considered statistically independent with random reflection coefficients, the standard deviation of which depends on the reflector material type based on empirical data on surface elements. Note that, in particular, small inhomogeneities and roughnesses of terrain are excluded from the geometric model of the surface and are taken into account due to the use of diffuse backscattering rather than mirror backscattering.

\subsection{Calculation of a set of facet parameters}

The simulation of the reflected signal begins with the sequential calculation of the slant range, Doppler frequency shift and power for each elementary reflector or surface facet.

The slant range is the first base parameter for any radar system that is directly related to the resolution and use of the radar. It is the distance from the radar antenna to the target (or reflector or facet) position. At any time, it can simply be determined from the descriptive geometry of the model shown in Fig. 2. Thus, according with relative coordinates $\left(x_{i}, y_{i}, z_{i}\right)$ of the $i^{\text {th }}$ facet along each of the 3 axes, the current value of the slant range is determined as:

$$
R_{i}=\sqrt{x_{i}^{2}+\left(H-y_{i}\right)^{2}+z_{i}^{2}}
$$


where $H$ is the radar antenna altitude relative the average surface level.

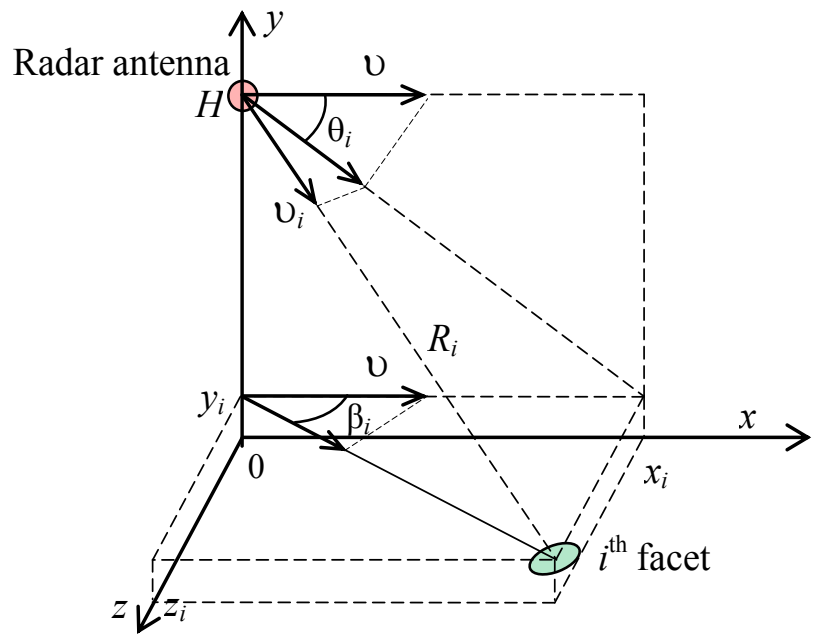

Fig. 2. Geometry of the relative positions of the radar antenna and the sample facet.

The Doppler frequency shift is the second base parameter for a radar system. Its use is extremely useful to increase radar application. Its definition is a key property for all imaging radars that calculates the images of the underlying surface using special signal processing $[1,7,8]$. Given the geometry of the model shown in Fig. 2, the Doppler frequency shift of the signal reflected from the facet is calculated by the expression:

$$
\Delta f_{i}=\frac{2 v_{i}}{\lambda}=\frac{2 v \cos \left(\theta_{i}\right) \cos \left(\beta_{i}\right)}{\lambda}
$$

where $v_{i}$ is the radial velocity of the radar platform in the direction of the $i^{\text {th }}$ facet; $\theta_{i}$ and $\beta_{i}$ are the angles for taking into account the individual direction of the facet (from the radar antenna, relative to the velocity vector $v$, which is also shown for the $i^{\text {th }}$ facet in Fig. 2) in the horizontal and vertical planes.

The power of the signal reflected from the target is the third base parameter for a radar system. In the case of terrain modeling using the facet model, any facet is a small target. Thus, the reflected facet power is in accordance with the radar equation, which can be written as the following expression:

$$
P_{i}=\frac{P_{0} \lambda^{2}}{(4 \pi)^{3}} \frac{\left(G_{\text {ant }} K_{i}\right)^{2}\left(G_{\text {surf }} B_{i}\right) S_{i}}{R_{i}^{4}} \eta_{i}
$$

where $P_{0}$ is the radar transmitter power; $\lambda$ is the carrier wavelength; $G_{a n t}$ is the antenna gain; $K_{i}$ is the coefficient of accounting of the antenna pattern; $G_{\text {surf }}$ is the reflection coefficient to account for the specific surface or target radar cross section (RCS); $B_{i}$ is the coefficient of the backscattering pattern; $S_{i}$ is the facet area; $\eta_{i}$ is the coefficient of accounting for losses in the distribution medium (for example, including shading of the rain drops or vegetation layer, if present).

To summarize, we can say that the structure of the intrinsic and calculated parameters of the facet of the universal mathematical model should include the above $(\cdot)_{i}$ parameters in order to take into account the possibilities of representing various types of landscape and artificial objects. Some additional parameters for representing natural surfaces will be discussed below. 


\subsection{Algorithm for modeling the reflected signal}

As already mentioned, the most common methods of digital modeling of radar signals reflected from complex surfaces and targets are based on facet models. In this case, the entire reflecting surface is divided into some elementary reflecting areas, i.e. facets, and then the signals reflected from each facet as from a point object are calculated.

This approach allows one to include various inhomogeneities in the surface that directly affect the nature of the reflected signal. Obviously, it is possible to combine facets with various properties to create complex phono-target environments, and with the dynamics, for example, in a form of a combat area model [7-9].

However, at the first stages of the study of the characteristics of signal processing algorithms in the radar, it is often enough to be limited only to a certain area of space where the concentrated, surface- or volume-distributed target is located. In this case, it is convenient to use a model capable of presenting the background-target environment with sufficient accuracy and with a given detail.

The proposed top-level of the algorithm for modeling the raw signal, i.e. signal reflected from the surface, is shown in Fig. 3.

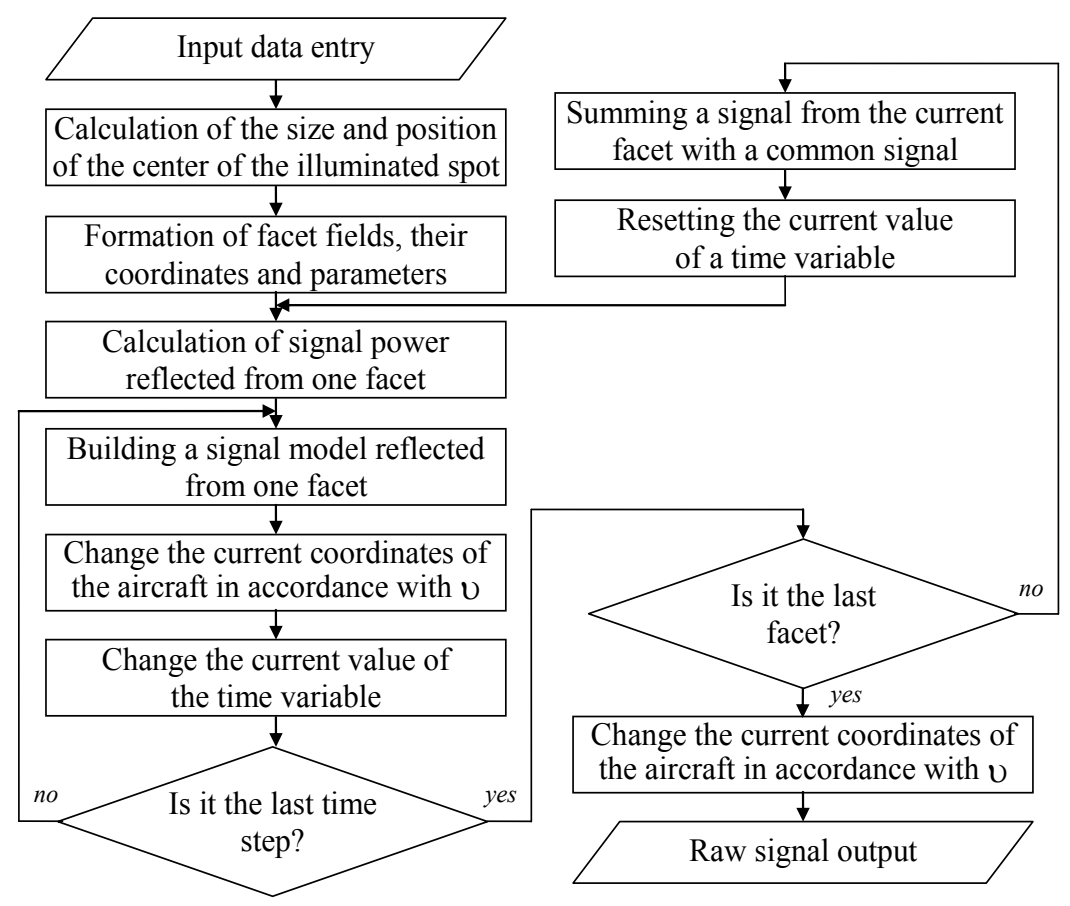

Fig. 3. Algorithm for modeling the raw signal reflected from the surface.

Also, for adequate signal modeling, it is necessary to take into account the initial data of the trajectory of the aircraft, radar, such as the altitude and components of the aircraft's flight speed vector, the angles of evolution of the aircraft (roll and pitch), the parameters of the radiation patterns of the receiving and transmitting elements of the antenna system (the width and shape of the radiation patterns), angles of deviation of the antenna axes from the vertical axis of the aircraft, the carrier frequency of the transmitter, the length of the probe pulse, the duty cycle of the pulses and the number of pulses emitted on the simulation path (for a pulse radar ), frequency deviation, modulation period and the number of modulation periods on the modeling path (for continuous-wave radars), signal sampling frequency. 


\section{Some exaples of mathematical modeling}

The objects of research were the following types of terrain: 1) a homogeneous rough surface, 2) a sea surface, 3) a forest surface.

For an accurate calculation of the energy distribution in the spectrum, a threedimensional mathematical model of the "radar - underlying surface - radar" channel is necessary, taking into account both the principles of radar signal processing, as well as the parameters of the underlying surface, velocity and evolution of the aircraft $[8,9]$.

For the greatest versatility, modeling of reflective underlying surfaces is carried out by creating a facet field consisting of elementary point reflectors. The facet coordinates can be set in various ways: randomly, in a square or rectangular lattice, in a hexagonal or triangular lattice, along concentric circles, in a spiral, etc. The number of faces must match the radar resolution for the range and angular coordinates.

\subsection{Homogeneous rough surface model}

Due to the fact that in the general case, for a homogeneous rough surface, the position of all reflectors is random, such a model makes it possible to take into account any (typical) radar situation in a simplified form. Its use is effective in the statistical study of reflected signals with an unknown (arbitrary) type of surface. The coordinates of the facet arrangement in the plane are random variables uniformly distributed within the irradiation spot. The vertical coordinate has a normal distribution, the dispersion of this value is set in advance and is an indicator of large surface roughness.

An example of such a facet field for nadir irradiation for a height of $100 \mathrm{~m}$, the size of large relief irregularities is $2.5 \mathrm{~m}$, the number of facets is 1000 , and with an irradiation spot radius of $50 \mathrm{~m}$, is shown in Fig. 4.

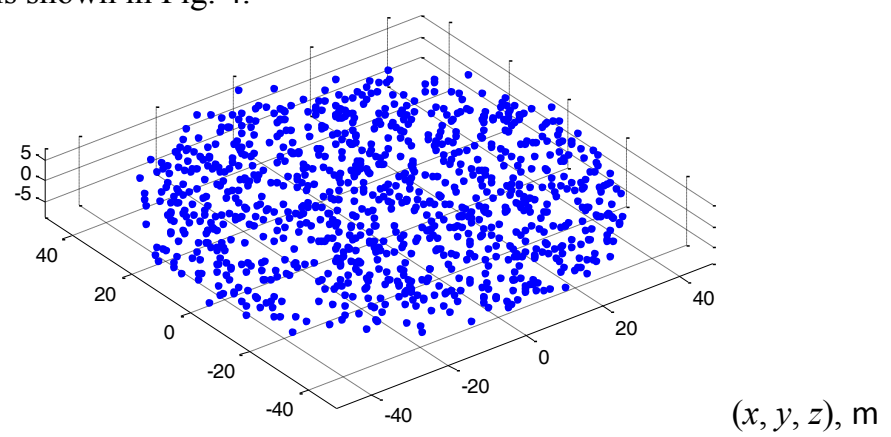

Fig. 4. Facet field of modeling reflectors of a homogeneous rough underlying surface.

\subsection{Sea model}

When studying the sea surface, it is necessary to take into account factors influencing the wave formation of the sea, such as: speed and direction of the wind, the length of the region of acceleration of waves, ocean movements, etc.

The sea surface modeling algorithm can account the model of the energy spectrum of sea waves (capillary for ripples, TMA for wind waves), the parameters for each elementary wave in the wave composition (height and wavelength, direction of propagation, wave phase, etc.) which are determined from this spectrum [6]. But in simple case (howewer, useful to study) it can be a one harmonic wave which moves in the desired direction.

Examples of a complex model and such a simplified facet field for a sea wave height of $7 \mathrm{~m}$, the speed of the wave along the $\mathrm{Z}$ axis is $7 \mathrm{~m} / \mathrm{s}$, along the $\mathrm{Y}$ axis is $4 \mathrm{~m} / \mathrm{s}$, the number of facets is 2000, and with an irradiation spot radius of $50 \mathrm{~m}$, is shown in Fig. 5. 

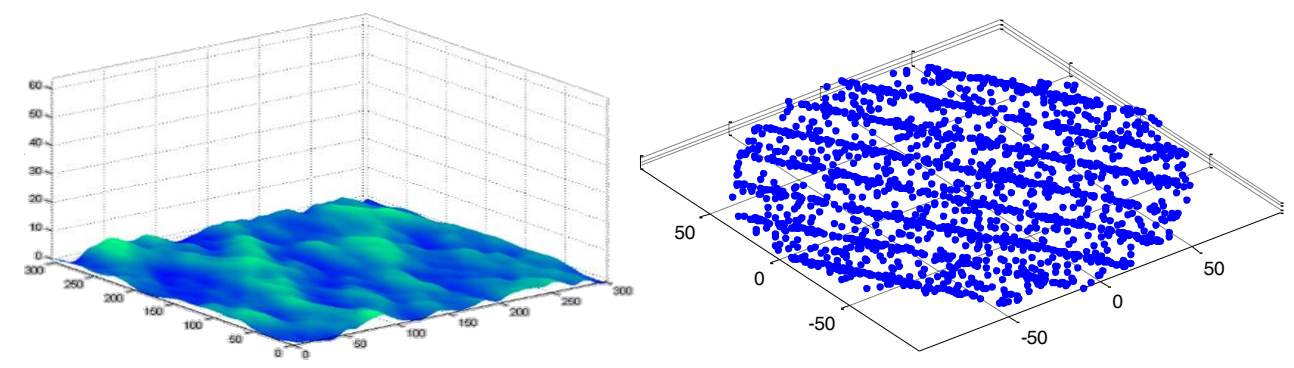

$(x, y, z), \mathrm{m}$

Fig. 5. Complex model and simplified facet field for modeling sea surface reflectors.

\subsection{Forest model}

It was experimentally established that in the centimetre wave band the signal from the canopy is comparable in power with the signal from the underlying soil (ground) [4-6, 9], and, with a resolution of $1 \mathrm{~m}$ or less, it is well localized, both in the presence of foliage and in the absence of it. Although, in a number of works, the forest surface is assigned to surface-distributed targets [2, 3], it is more correct to consider it layered or volumedistributed, because forest canopy as a radar target has a distribution both on the surface and in depth. The signal reflected from it is more "blurred" in range than the signal reflected from other types of underlying surfaces.

Forest surface is a complex heterogeneous surface that can be divided with some simplification into three layers: soil, undergrowth, trees (trunk, branches, leaves). During the modeling process the first stage, the soil layer is set, which is a plane with a small topography, the boundaries and area of which depend on the aircraft height and antenna pattern for a given aircraft motion path. Then, the layered structure for the undergrowth and tree canopy is simulated.

Examples of a complex model [9] and such a simplified forest facet field for the first tree level with height of trees of about of $30 \mathrm{~m}$ (number of facets is 220), with the small tree's height of $10 \mathrm{~m}$ (number of facets is 100), with the number of facets is 1000 for the ground level with the size of large relief irregularities is $2.5 \mathrm{~m}$, is shown in Fig. 6.
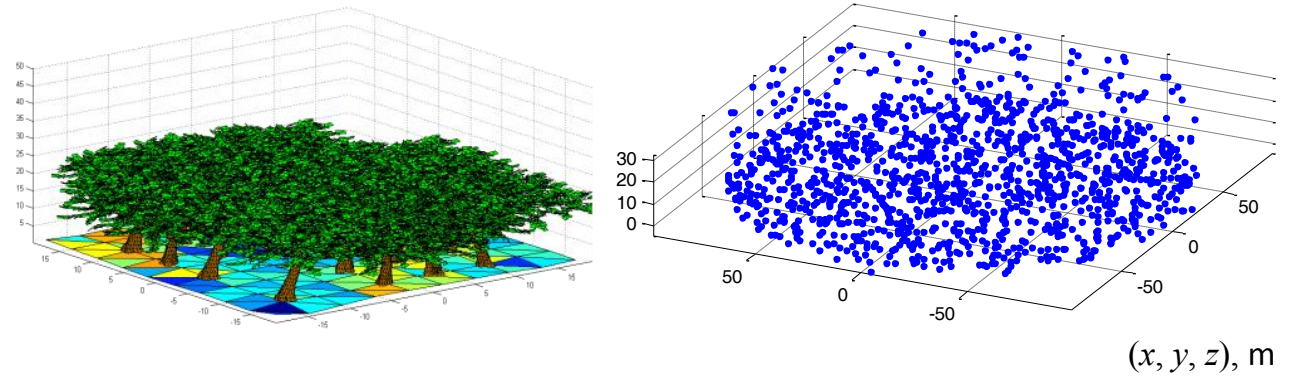

Fig. 6. Complex model and simplified facet field for modeling forest reflectors.

Unfortunately, it is difficult to implement fast and complete complex models for sea and forest surfaces. The choice of a model is largely determined by the availability of sufficient computing resources, that is why we are currently concentrating on some simplifications [9]. However, the proposed simplified facet models produce the desired level of quality for generated raw signals [10], because these signals include all the basic surface parameters that are needed for various radar systems, including SAR. 


\section{Conclusion}

In this paper the algorithm that uses a phenomenological model to describe underlying surfaces was developed. The universality of the considered model of radar signals lies in the possibility of reducing the amount of calculations by using the model of point reflectors while maintaining the main features of the reflected signal formation as the sum of the reflected signals, taking into account the amplitude and phase ratios of the partial signals. Such a simplification is correct from the point of view of developing and debugging algorithms for joint work of the formation and processing models (determining the relative range/altitude, synthesizing radar images) of the signals reflected from various types of natural and humanmade objects when modeling and studying the characteristics, resolution, relative power budgets of various radar and altimeter systems, including SAR.

It is worthwhile to note, that mathematical modeling of radar system operation is the main way to study common and state-of-the-art methods of radar signal processing, as well as to develop new, more efficient, reliable and accuracy methods of radiovision of various vehicles.

\section{Acknowledgments}

This research has been funded by a grant of the Ministry of Science and Higher Education of the Russian Federation (project No. 8.2538.2017/4.6).

\section{References}

1. B. R. Mahafza, Radar Systems Analysis and Design Using MATLAB (Chapman and Hall/CRC, 2013)

2. A. I. Baskakov, T. S. Zhutyayeva, Yu. I. Lukashenko, Locational Methods for Studying Objects and Media (in Russian, Academy, Moscow, 2011)

3. S. G. Zubkovich, Statistical characteristics of radio signals reflected from the Earth's surface (in Russian, Soviet radio, Moscow, 1968)

4. P. Liang, M. Moghaddam, L. E. Pierce, R. M. Lucas, IEEE Transactions on geoscience and remote sensing, 43, no. 11, 2612-2626 (2005).

5. F. T. Ulaby, M. C. Dobson, Handbook of radar scattering statistic for terrain (Artech house, USA, 1989)

6. A. K. Fung, K. S. Chen, Microwave Scattering and Emission Models for Users (Artech house, USA, 2010)

7. V. G. Kobernichenko, Radioelectronic Earth `s remote sensing systems (in Russian, UrFU, Yekaterinburg, 2016)

8. G. S. Kondratenkov, A. U. Frolov, Radiovision. Radar Earth 's remote sensing systems (in Russian, Radiotechnica, Moscow, 2005)

9. A. S. Bokov, A. E. Smertin, V. G. Vazhenin, CEUR-WS, 2274, 42-51 (2018).

10. Yu. V. Markov, A. S. Bokov, V. G. Vazhenin, S. A. Margilevsky, RSEMW-2019, 352-355 (2019) 